\title{
Vorapaxar: The Current Role and Future Directions of a Novel Protease-Activated Receptor Antagonist for Risk Reduction in Atherosclerotic Disease
}

\author{
Rebecca J. Gryka' ${ }^{1}$ Leo F. Buckley ${ }^{2} \cdot$ Sarah M. Anderson $^{3}$
}

Published online: 6 January 2017

(c) The Author(s) 2017. This article is published with open access at Springerlink.com

\begin{abstract}
Introduction Despite the current standard of care, patients with cardiovascular disease remain at a high risk for recurrent events. Inhibition of thrombin-mediated platelet activation through protease-activated receptor-1 antagonism may provide reductions in atherosclerotic disease beyond those achievable with the current standard of care. Objective Our primary objective is to evaluate the clinical literature regarding the role of vorapaxar (Zontivity ${ }^{\mathrm{TM}}$ ) in the reduction of cardiovascular events in patients with a history of myocardial infarction and peripheral artery disease. In particular, we focus on the potential future directions for protease-activating receptor antagonists in the treatment of a broad range of atherosclerotic diseases.

Data Sources A literature search of PubMed and EBSCO was conducted to identify randomized clinical trials from August 2005 to June 2016 using the search terms: "vorapaxar', 'SCH 530348', 'protease-activated receptor-1 antagonist', and 'Zontivity $^{\mathrm{TM}}$, . Bibliographies were searched and additional resources were obtained.
\end{abstract}

Electronic supplementary material The online version of this article (doi:10.1007/s40268-016-0158-4) contains supplementary material, which is available to authorized users.

Rebecca J. Gryka

rgryka@cedarville.edu

1 Pharmaceutical Sciences Department, School of Pharmacy, Cedarville University, 251 North Main Street, Cedarville, OH 45314, USA

2 Department of Pharmacotherapy and Outcomes Science, School of Pharmacy, Virginia Commonwealth University, Cedarville, OH, USA

3 Center for Pharmacy Care, Duquesne University, Pittsburgh, PA, USA
Results Vorapaxar is a first-in-class, protease-activated receptor-1 antagonist. The Thrombin Receptor Antagonist for Clinical Event Reduction (TRACER) trial did not demonstrate a significant reduction in a broad primary composite endpoint. However, the Thrombin-Receptor Antagonist in Secondary Prevention of Atherothrombotic Ischemic Events (TRA $2^{\circ} \mathrm{P}-\mathrm{TIMI} 50$ ) trial examined a more traditional composite endpoint and found a significant benefit with vorapaxar. Vorapaxar significantly increased bleeding compared with standard care. Ongoing trials will help define the role of vorapaxar in patients with peripheral arterial disease, patients with diabetes mellitus, and other important subgroups. The use of multivariate modeling may enable the identification of subgroups with maximal benefit and minimal harm from vorapaxar.

Conclusion Vorapaxar provides clinicians with a novel mechanism of action to further reduce the burden of ischemic heart disease. Identification of patients with a high ischemic risk and low bleeding risk would enable clinicians to maximize the utility of this unique agent.

\section{Key Points}

Vorapaxar is a novel, first-in-class, proteaseactivated receptor-1 antagonist.

Vorapaxar may provide incremental protection against thrombotic cardiovascular events beyond the standard of care.

Ongoing studies will help define the ideal patient populations for protease-activated receptor antagonism. 


\section{Introduction}

Cardiovascular diseases (CVDs) represent a significant global public health problem. According to the World Health Organization, CVDs are the world's leading cause of death and disability. Currently, 17 million deaths are attributable to cardiovascular events (CVEs) annually [1]. Of those, over $75 \%$ have atherothrombosis as an underlying pathophysiology: 7.3 million due to ischemic heart disease and 6.2 million due to strokes. Even with early revascularization and potent dual antiplatelet therapy, residual mortality remains high [2]. As a result, assessment of new antiplatelet agents is an expanding research area.

Platelets play a major role in primary hemostasis, vascular repair, and formation of pathogenic thrombi. Inhibition of platelet activation by aspirin and adenosine diphosphate (ADP) receptor antagonists decreases platelet aggregation and thus decreases CVEs (Online Fig. 1) [3]. These agents show long-term benefits in secondary prevention but are associated with increased bleeding, and the rate of recurrent ischemic events remains high. Targeting the protease-activated receptor-1 (PAR-1) found on human platelets provides a promising new mechanism to block platelet activation and decrease the residual risk of CVEs.

Vorapaxar, a first in its class, is an orally available PAR-1 antagonist approved for the reduction of CVEs in patients with a history of myocardial infarction (MI) or with peripheral arterial disease (PAD). Vorapaxar reduced the rate of the combined endpoint of cardiovascular death, stroke, and $\mathrm{MI}$ in one of two phase III trials $[4,5]$. However, coupled to this reduction in the primary endpoint is the increased risk of bleeding. This article outlines the effects of vorapaxar on ischemic and bleeding outcomes as well as its potentially novel roles in the treatment of atherosclerotic disease.

\section{Data Sources}

A literature search of PubMed, IPA, OneSearch, and MEDLINE was conducted from June 2008 to June 2016. The search terms 'vorapaxar', 'SCH 530348', 'protease-activated receptor-1 antagonist', and 'Zontivity ${ }^{\mathrm{TM}}$, were used. Studies published in English evaluating vorapaxar were reviewed with an emphasis on randomized controlled trials. Citations from available articles were retrieved for additional references.

\section{Pharmacology}

Thrombin, a serine protease, facilitates hemostasis via multiple actions including platelet activation, protein $\mathrm{C}$ activation, and conversion of fibrinogen to fibrin. Thrombin is the most potent circulating activator of platelets and mediates these effects primarily through activation of the G-protein-coupled PAR-1 on the platelet surface [6-8]. Platelet activation through PAR-1 signaling results in extracellular ADP release, which then acts in an autocrine fashion to activate platelet ADP receptors, sustaining longterm effects [9].

Vorapaxar (SCH50348) is a potent, oral, reversible thrombin receptor antagonist, which selectively antagonizes the PAR-1 to prevent thrombin-mediated platelet activation (Online Fig. 1). Selective PAR-1 blockade by vorapaxar results in inhibition of thrombin-induced platelet aggregation but does not appear to impact primary hemostatic functions, including the formation of fibrin to stabilize the platelet plug $[10,11]$.

\section{Pharmacokinetics}

In patients with non-ST-segment elevation acute coronary syndromes (NSTE-ACS), vorapaxar rapidly achieves $\geq 80 \%$ inhibition of thrombin receptor-activating, peptideinduced platelet aggregation by $2 \mathrm{~h}[12,13]$. Owing to its long half-life of 173-269 h, steady-state concentrations of vorapaxar are reached approximately 21 days after initial dosing and platelet inhibition is sustained for $>4$ months.

Vorapaxar pharmacokinetic parameters are unaffected by food consumption [14, 15]. No dosage adjustment is needed for patients with end-stage renal failure [16]. While no dosage adjustment is recommended for mild to moderate hepatic impairment, vorapaxar is not recommended in patients with severe hepatic failure owing to the increased inherent risk of bleeding in these patients [17, 18]. Vorapaxar is hepatically metabolized by cytochrome $\mathrm{P} 450$ (CYP)3A4 and CYP2J2 enzymes, resulting in the formation of an active metabolite with equal potency to the parent compound [19]. Vorapaxar is a weak inhibitor of intestinal p-glycoprotein [20,21]. Table 1 shows additional pharmacokinetic parameters.

\section{Drug Interactions}

Pharmacokinetic drug interaction studies are few with vorapaxar; however, because vorapaxar is metabolized by CYP3A4 (alongside CYP2J2), drugs that are inducers or inhibitors of CYP3A4 will potentially impact the elimination of vorapaxar. Co-administration of ketoconazole for 3 weeks increased vorapaxar exposure by twofold while co-administration with rifampin reduced vorapaxar exposure by $50 \%$ [22]. Thus, strong inducers or inhibitors of CYP3A4 should be avoided. Additionally, dose adjustments appear to be unnecessary for patients on CYP2C9/ 
Table 1 Pharmacokinetic parameters $^{\mathrm{a}}$

\begin{tabular}{|c|c|c|}
\hline & Fasted & $\mathrm{Fed}^{\mathrm{b}}$ \\
\hline \multicolumn{3}{|l|}{ Absorption [13] } \\
\hline Mean $C_{\max }$ & $23.7 \mathrm{ng} / \mathrm{mL}$ & $19.4 \mathrm{ng} / \mathrm{mL}$ \\
\hline Mean $\mathrm{AUC}_{0-72 \mathrm{~h}}$ & $323 \mathrm{ng} \cdot \mathrm{h} / \mathrm{mL}$ & $313 \mathrm{ng} \cdot \mathrm{h} / \mathrm{mL}$ \\
\hline Median $T_{\max }$ & $1.25 \mathrm{~h}$ & $2.00 \mathrm{~h}$ \\
\hline \multicolumn{3}{|l|}{ Distribution $[15,19]$} \\
\hline Mean $V_{\mathrm{d}} / F$ & $508 \mathrm{~L}$ & \\
\hline Plasma protein binding & $\geq 99 \%$ & \\
\hline \multicolumn{3}{|l|}{ Metabolism $[18,19]$} \\
\hline \multicolumn{3}{|l|}{ Primary metabolites } \\
\hline \multicolumn{3}{|c|}{ Metabolites produced: M19 $\gg$ M20 } \\
\hline \multicolumn{3}{|c|}{ M19: inactive amine metabolite formed by carbamate cleavage } \\
\hline \multicolumn{3}{|c|}{ M20: hydroxylated metabolite equipotent with vorapaxar } \\
\hline \multicolumn{3}{|l|}{ Hepatic isoenzymes } \\
\hline \multicolumn{3}{|l|}{ CYP3A4 } \\
\hline \multicolumn{3}{|c|}{ Major CYP enzyme responsible for M19 formation } \\
\hline \multicolumn{3}{|c|}{ Primary metabolite of vorapaxar is the M19 metabolite ( $\approx 80 \%$ of exposure) } \\
\hline \multicolumn{3}{|l|}{ CYP2J2 and CYP3A4 } \\
\hline \multicolumn{3}{|c|}{ Responsible for the metabolism of vorapaxar to M20 } \\
\hline \multicolumn{3}{|c|}{ Only becomes detectable after long-term administration of vorapaxar } \\
\hline \multicolumn{3}{|l|}{ Elimination } \\
\hline \multicolumn{3}{|c|}{ Metabolites eliminated primarily in feces and, to a much smaller extent, urine } \\
\hline \multicolumn{3}{|c|}{ No unchanged vorapaxar detectable in urine } \\
\hline \multicolumn{3}{|l|}{ Excretion } \\
\hline Mean CL/F [15] & $1.66 \mathrm{~L} / \mathrm{h}$ & \\
\hline Mean half-life [12] & $173-269 \mathrm{~h}$ & \\
\hline \multicolumn{3}{|c|}{$\begin{array}{l}A U C_{0-72} h \text { area under the plasma concentration-time curve from time } 0 \text { to } 72 \mathrm{~h}, C L / F \text { apparent total body } \\
\text { clearance, } C_{\max } \text { maximum plasma concentration, } C Y P \text { cytochrome } \mathrm{P} 450, T_{\max } \text { time to maximum plasma } \\
\text { concentration, } V_{d} / F \text { apparent volume of distribution }\end{array}$} \\
\hline b Fed state after a high-fat & 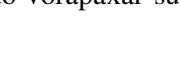 & \\
\hline
\end{tabular}

2C19 substrates [18]. It was determined that vorapaxar is only a weak inhibitor of $p$-glycoprotein as evidenced by the fact that vorapaxar had no impact on serum digoxin concentrations. Thus, no dose adjustment of digoxin is necessary [21]. Concomitant use of vorapaxar and platelet glycoprotein IIb-IIIa inhibitors should be limited because of the potential for a pharmacodynamic drug interaction, resulting in a heightened predisposition to bleeding [23].

\section{Current Use of Vorapaxar}

Vorapaxar is approved for the reduction of thrombotic CVEs in patients with a history of MI or with PAD. The current clinical use of vorapaxar is based on two large, randomized clinical trials conducted in patients with a broad range of vascular diseases. At present, vorapaxar has limited clinical utility. Multivariate analyses will be required to identify features of patients with a high ischemic risk and low bleeding risk in whom vorapaxar presents a net clinical benefit.

\section{Clinical Trials Overview}

The Thrombin Receptor Antagonist for Clinical Event Reduction (TRACER) trial was a randomized, doubleblind, multinational trial comparing vorapaxar (40-mg loading dose followed by $2.08 \mathrm{mg}$ daily) with placebo, on a background of standard antiplatelet therapy, in 12,944 patients with NSTE-ACS (Table 2) [4, 24].

The primary efficacy endpoint of death from cardiovascular causes, MI, stroke, recurrent ischemia with hospitalization, or urgent coronary revascularization occurred in 1031 of $6473(18.5 \%)$ patients taking vorapaxar and in 1102 of $6371(19.9 \%)$ patients receiving placebo (hazard ratio [HR] 0.92; 95\% confidence interval [CI] 0.85-1.01; $p=0.07)$. Vorapaxar did reduce the composite endpoint of 
Table 2 Phase III vorapaxar clinical trials

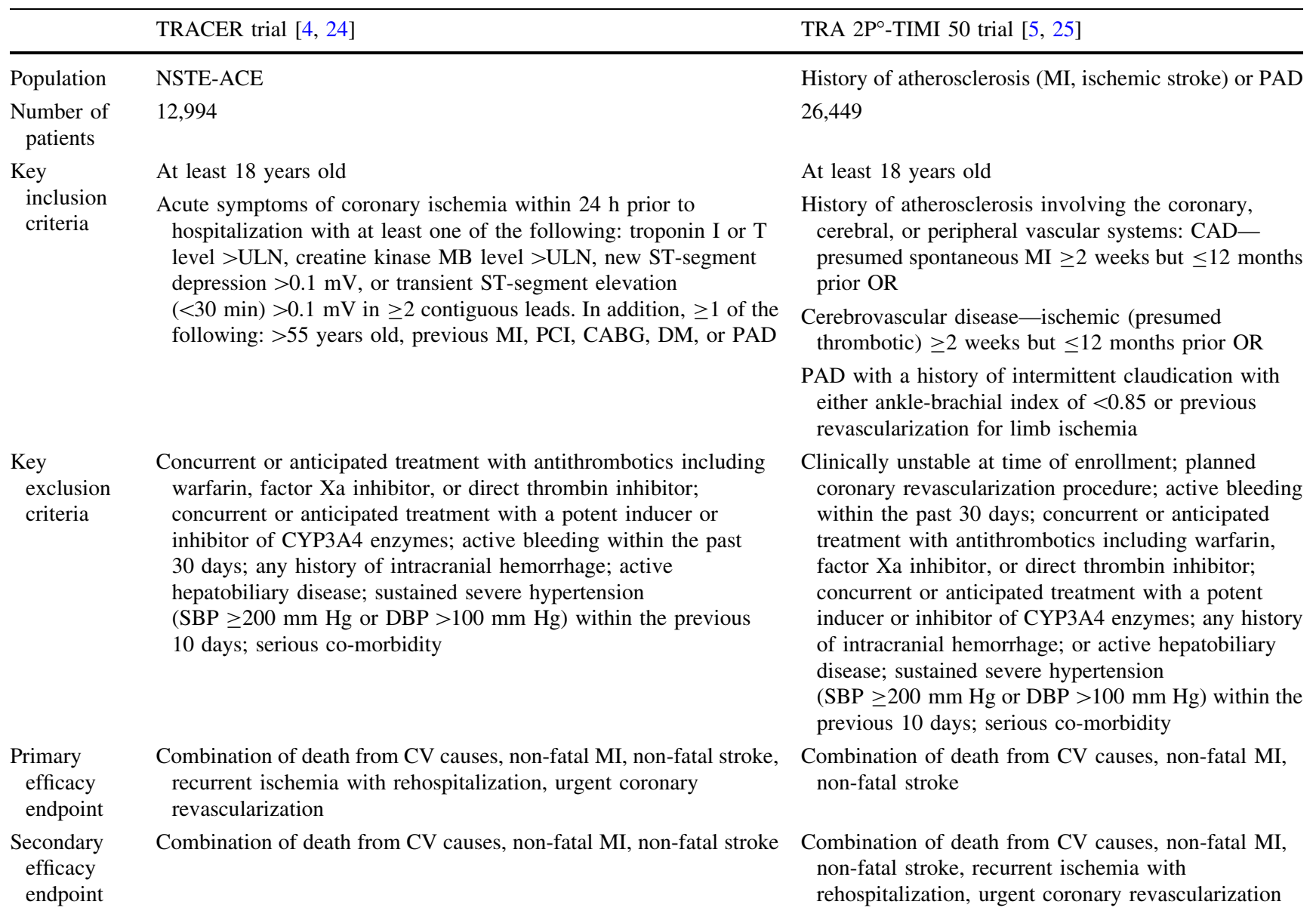

$C A B G$ coronary artery bypass grafting, $C A D$ coronary artery disease, creatine kinase $M B$ cardiac isozyme of creatine kinase, $C V$ cardiovascular, $C Y P$ cytochrome P450, DBP diastolic blood pressure, $D M$ diabetes mellitus, $H g$ mercury, $M I$ myocardial infarction, $N S T E$ - $A C E$ non-ST-segment elevation acute coronary syndrome, $P A D$ peripheral artery disease, $P C I$ percutaneous coronary intervention, $S B P$ systolic blood pressure, TRACER Thrombin Receptor Antagonist for Clinical Event Reduction, TRA $2^{\circ} \mathrm{P}-T I M I 50$ Thrombin-Receptor Antagonist in Secondary Prevention of Atherothrombotic Ischemic Events, $U N L$ upper limit of normal

death from cardiovascular causes, MI, or stroke compared with placebo (16.4 vs. $14.7 \%$; HR 0.89 ; 95\% CI 0.81-0.98; $p=0.02$ ). However, cautious interpretation of this secondary endpoint is warranted in light of the non-significant difference in the primary endpoint.

Vorapaxar, in comparison with placebo, was associated with a significantly increased risk of bleeding when added to aspirin and a $\mathrm{P} 2 \mathrm{Y}_{12}$ inhibitor (GUSTO combined moderate and severe bleeding: $7.2 \%$ vorapaxar and $5.2 \%$ placebo, $p<0.001$; TIMI clinically significant bleeding: 20.2 vs. $11.7 \%, p<0.001)$. Importantly, intracranial bleeding was increased fivefold in vorapaxar-treated patients compared with placebo $(1.1$ vs. $0.2 \%, p<0.001)$. As a result of this increased risk of intracranial bleeding, an interim safety review in 2011 recommended discontinuation of the trial 6 months prior to the originally planned end date.

The Thrombin-Receptor Antagonist in Secondary Prevention of Atherothrombotic Ischemic Events (TRA $\left.2^{\circ} \mathrm{P}-\mathrm{TIMI} 50\right)$ trial was a phase III, randomized, doubleblind, placebo-controlled, multinational clinical trial created to evaluate the safety and efficacy of vorapaxar in secondary prevention (Table 2) [5, 25]. There are several important differences between TRACER and TRA $2^{\circ} \mathrm{P}$ TIMI 50. While TRACER enrolled patients during the acute phase, TRA $2^{\circ} \mathrm{P}-\mathrm{TIMI} 50$ enrolled 26,449 patients receiving standard care for a broad range of CVDs, including coronary, cerebrovascular, and peripheral disease. Another notable difference between these two large phase III trials is the study treatment dosing, as the loading dose used in TRACER was omitted in TRA $2^{\circ} \mathrm{P}$-TIMI 50. Last, the primary endpoint in TRA $2^{\circ} \mathrm{P}-\mathrm{TIMI} 50$ was truncated to the composite of cardiovascular death, nonfatal MI, or nonfatal stroke and did not include recurrent ischemia with re-hospitalization or urgent coronary revascularization. Once the increased risk of intracranial bleeding became apparent in the TRACER trial, study 
treatment was discontinued in all TRA $2^{\circ} \mathrm{P}$-TIMI 50 patients with a prior stroke or transient ischemic attack.

Like TRACER, TRA $2^{\circ} \mathrm{P}$-TIMI 50 found a significant reduction in the composite of cardiovascular death, nonfatal MI, or nonfatal stroke with vorapaxar treatment $(9.3$ vs. $10.5 \%$; HR 0.87 ; 95\% CI 0.80-0.94; $p<0.001$ ) [5, 25]. However, this risk reduction was accompanied by an increased risk of GUSTO moderate or severe bleeding (4.2 vs. $2.5 \%$ ), TIMI clinically significant bleeding (15.8 vs. $11.1 \%)$, and intracranial hemorrhage (1.0 vs. $0.5 \%)$ in the vorapaxar group ( $p<0.001$ for all three assessments).

Together, TRACER and TRA $2^{\circ}$ P-TIMI 50 demonstrate that vorapaxar, in addition to standard antiplatelet therapy, provides an incremental risk reduction by targeting a novel platelet activation pathway. These two trials also demonstrate that triple antiplatelet therapy is accompanied by a significant increase in the risk of bleeding. While the goals of TRACER and TRA $2^{\circ} \mathrm{P}-\mathrm{TIMI} 50$ were to demonstrate the benefits of PAR-1 antagonism in a wide range of patients, a net clinical benefit is unlikely to be achieved in the majority of patients owing to the significant bleeding risk of vorapaxar. However, subsequent re-analysis of well curated data from these robust clinical trials has provided insight into subgroups of patients whose risk of atherosclerotic disease justifies the risk of bleeding (diabetes mellitus) or who lack effective treatment options without vorapaxar (PAD). Interestingly, vorapaxar was studied in the NSTE-ACS population, which is more heterogeneous and at an overall lower risk than the STEACS population. Furthermore, head-to-head comparisons between vorapaxar and more potent $\mathrm{P} 2 \mathrm{Y}_{12}$ antagonists would be of considerable interest.

\section{Future Directions: Potential Clinical Use of Vorapaxar}

Although the development of another PAR-1 antagonist, atopaxar, has been discontinued, the TRACER and TRA $2{ }^{\circ} \mathrm{P}$-TIMI 50 studies support a role for vorapaxar in a select group of patients. Preliminary data suggest that multivariable modeling can be used to identify patients at a high ischemic risk and low bleeding risk [26]. A meta-analysis which combined phase II and III clinical trials investigated the overall clinical effects of vorapaxar [27]. Overall, reductions in MI were offset by a doubling in the risk of intracranial hemorrhage. There was no significant difference in TIMI major bleeding or cardiovascular mortality.

However, several questions remain to be answered. There is limited evidence on triple therapy with vorapaxar in addition to aspirin and the more potent platelet antagonists, prasugrel and ticagrelor. Because of the significant potential for bleeding, this combination therapy should be avoided with few exceptions. Similarly, whether PAR-1 antagonism provides benefit in addition to extremely potent ADP receptor inhibition is unknown. An ongoing pharmacodynamic trial of 126 patients will provide initial data on this combination of platelet antagonists [28].

Although TRA $2^{\circ} \mathrm{P}$-TIMI 50 demonstrated a significant benefit in the reduction of recurrent ischemic events, the risk of bleeding necessitates careful identification of candidates for this very potent platelet inhibitor. The inclusion of a heterogeneous cohort of coronary, cerebrovascular, and PADs necessitates "teasing out", which subgroups derive the most benefit and least harm with vorapaxar treatment. In this section, we review the TRACER and TRA $2{ }^{\circ} \mathrm{P}-$ TIMI 50 to search for answers to these important questions.

\subsection{Peripheral Artery Disease}

Patients with PAD are not only at an increased risk for acute limb-threatening events, but also for systemic manifestations of atherosclerotic disease, such as cardiovascular death, MI, and ischemic stroke [29, 30]. In the TRA $2^{\circ} \mathrm{P}$-TIMI 50 trial, 3787 patients were diagnosed with PAD, defined as a history of claudication and an ankle-brachial index of $<0.85$ or prior revascularization for limb ischemia. Although the primary composite endpoint (which included coronary, cerebrovascular, and peripheral components) was not significantly reduced in patients with PAD taking vorapaxar, hospitalization as a result of acute limb ischemia and peripheral artery revascularization was significantly reduced by vorapaxar $(2.3 \%$ vorapaxar vs. $3.9 \%$ placebo; $p=0.006$ and $18.4 \%$ vorapaxar vs. $22.2 \%$ placebo; $p=0.017$, respectively). This benefit was offset by a significant increase in the risk of bleeding with vorapaxar (7.4\% vorapaxar vs. $4.5 \%$ placebo; $p=0.001$ ) [30].

The TRACER trial did not purposefully target patients with PAD and only 936 (7.2\%) such patients were randomized. While the event rates between vorapaxar and placebo were not significantly different owing to the small sample size, the overall profile of vorapaxar was similar to that found in TRA $2^{\circ} \mathrm{P}$-TIMI 50: reduced peripheral revascularization procedures and a lower extremity amputation rate $(8.1$ vs. $9.0 \% ; p=0.158$ and 0.9 vs. $1.5 \%$; $p=0.107$, respectively). Similarly, GUSTO moderate/severe bleeding was increased with vorapaxar use in patients with PAD (HR 1.47, 95\% CI 0.89-2.45) but not to a greater extent than patients without PAD ( $p$-interaction $=0.921)$ [31]. These results indicate that vorapaxar may have a role in the treatment of PAD, given the lack of effective therapies available. Although a small phase IV trial is underway to evaluate vorapaxar in this setting, a prospective study is likely required for vorapaxar to gain an indication specifically for the prevention of acute limb ischemia or peripheral revascularization [32]. 


\subsection{Diabetes Mellitus}

Patients with diabetes, owing to their increased risk of CVEs, may benefit from additional platelet inhibition with vorapaxar to a greater extent than non-diabetic patients. In diabetic patients $(n=3623)$ in TRA $2^{\circ} \mathrm{P}$-TIMI 50 , the primary endpoint of cardiovascular death, MI, or cerebrovascular accident occurred more frequently than in nondiabetic patients $(n=16,896)$ regardless of randomization (14.1 vs. $7.4 \% ; p<0.001)$ [33, 34]. However, diabetic patients significantly benefited from additional antiplatelet therapy with vorapaxar with respect to the primary composite endpoint (12.6 vs. $15.7 \% ; p=0.004)$. GUSTO moderate to severe bleeding was increased in diabetic patients with vorapaxar vs. placebo (4.7 vs. $2.8 \%$, HR 1.59 , $95 \%$ CI $1.09-2.32 ; p=0.02$ ). The risk of GUSTO moderate to severe bleeding was not different with vorapaxar in diabetic and non-diabetic patients ( $p$-interaction $=0.95$ ). An ongoing phase IV trial is designed to assess the effects of vorapaxar in addition to dual antiplatelet therapy with aspirin and clopidogrel compared with just dual antiplatelet therapy alone in diabetic patients [35].

\subsection{Coronary Artery Bypass Grafting}

The increased risk of thrombotic complications following coronary artery bypass grafting (CABG) can be attributed to increased thrombin generation, which occurs both during the procedure and continues after the procedure [36]. Whellan et al. proposed that PAR-1 antagonism could reduce both graft occlusion and native coronary thrombosis after CABG [37]. Among 1312 patients undergoing CABG during the TRACER trial (vorapaxar, $n=639$ and placebo, $n=673$ ), vorapaxar significantly reduced ischemic events compared with placebo (HR 0.55, 95\% CI $0.36-0.83 ; p=0.005)$. Bleeding related to CABG during hospitalization did not differ significantly between vorapaxar and placebo (GUSTO moderate to severe bleeding: odds ratio $1.25,95 \%$ CI $0.93-1.68 ; p=0.13$; TIMI major bleeding: odds ratio $1.36,95 \%$ CI $0.92-2.02 ; p=0.12$ ) $[4,37]$. Hence, in patients with NSTE-ACS undergoing CABG, vorapaxar was associated with a significant decrease in ischemic events. Notably, clopidogrel was used in only $18 \%$ of patients undergoing CABG vs. $85 \%$ in nonCABG. It is possible that the increase in bleeding events was not seen in this subgroup owing to the infrequent use of clopidogrel.

\subsection{Stroke}

Vorapaxar is strictly contraindicated in patients with a history of stroke or transient ischemic attack because of a significant increase in the risk of intracranial bleeding.
Among TRA $2^{\circ} \mathrm{P}$-TIMI 50 patients with prior stroke, recurrent stroke was not reduced by vorapaxar $(10.1 \%$ vorapaxar vs. $7.5 \%$ placebo, HR 1.13 , 95\% CI $0.90-1.40$; $p=0.30$ ), and GUSTO moderate or severe bleeding was higher in the vorapaxar group (4.2\% vorapaxar vs. $2.4 \%$ placebo, HR 1.93, 95\% CI 1.33-2.79; $p<0.001)[5,38]$. Importantly, intracranial bleeding was significantly increased in the vorapaxar group, including subdural or epidural bleeding (2.5\% vorapaxar vs. $1.0 \%$ placebo; $p<0.001)$. Fatal bleeding was numerically higher for vorapaxar vs. placebo but not statistically significant ( 8 vs. $4 ; p=0.26$ ). However, a more recent study demonstrates that vorapaxar may provide effective stroke prevention in patients without a history of prior stroke [39].

\subsection{Background Antiplatelet Therapy}

Vorapaxar may have greater efficacy in patients treated with aspirin alone compared with aspirin plus a $\mathrm{P} 2 \mathrm{Y}_{12}$ inhibitor [4]. In TRACER patients treated with clopidogrel (91.8\% of all patients), the risk of GUSTO moderate or severe bleeding was increased significantly compared with those treated with aspirin alone (HR $0.95,95 \%$ CI $0.65-1.40$ with no thienopyridine and HR $1.45,95 \%$ CI $1.23-1.71$ with thienopyridine; $p$-interaction $=0.04$ ) [40, 41].

In TRA $2^{\circ} \mathrm{P}-\mathrm{TIMI} 50$, dual antiplatelet therapy was less common than in TRACER (58\% of all patients) [42]. Thienopyridines were intentionally used in 15,256 patients (58\%) overall. There was no significant difference in the reduction of the primary endpoint between vorapaxar with thienopyridine and vorapaxar with no thienopyridine ( $p$ interaction $=0.64)$. Compared with TRACER, GUSTO moderate to severe bleeding was increased with vorapaxar in TRA $2^{\circ} \mathrm{P}$-TIMI 50 , but there was no significant interaction based on current ( $p$-interaction $=0.29)$ or prior $(p$ interaction $=0.99$ ) thienopyridine use. Vorapaxar decreased the risk of CVD, MI, or stroke in stable patients with a history of CVD with or without thienopyridines [42, 43]. In both TRACER and TRA $2^{\circ}$ P-TIMI 50, few patients were receiving more potent $\mathrm{P}_{2} \mathrm{Y}_{12}$ inhibition with prasugrel or ticagrelor. The interaction between aspirin dose and the efficacy and safety profile of vorapaxar was investigated in both TRACER and TRA $2^{\circ}$ P-TIMI 50. Aspirin dose did not modify the efficacy or safety profile significantly in either study $[4,5]$.

Because vorapaxar has been documented to increase bleeding risks, healthcare professionals must be cognizant of a patient's general underlying bleeding risk. General risk factors for bleeding include older age, low body weight, reduced renal or hepatic function, history of bleeding disorders, and use of other drugs that impact the coagulation pathways. Vorapaxar should be used cautiously or not at all 
in these patients. The patients who have a high ischemic risk coupled with a low bleeding risk will benefit most from the addition of vorapaxar to standard care [26].

\section{Conclusion}

The residual risk of atherosclerotic events in patients with coronary, cerebrovascular, and PAD remains unacceptably high, even after revascularization and treatment with potent platelet ADP receptor antagonists. Vorapaxar, the only available PAR-1 antagonist, may provide clinicians with an effective treatment for patients with strong risk factors, such as diabetes. Similarly, vorapaxar may represent a novel approach to the treatment of PAD. The major limitation to vorapaxar therapy is bleeding, as expected with antiplatelet therapy. However, vorapaxar may meet the requirements of patients who need additional therapy to reduce the risk for CVEs.

\section{Compliance with Ethical Standards}

Funding The authors received no funding for the preparation of this manuscript.

Conflict of interest Rebecca J. Gryka, Leo F. Buckley, and Sarah M. Anderson have no conflicts of interest to report.

Open Access This article is distributed under the terms of the Creative Commons Attribution-NonCommercial 4.0 International License (http://creativecommons.org/licenses/by-nc/4.0/), which permits any noncommercial use, distribution, and reproduction in any medium, provided you give appropriate credit to the original author(s) and the source, provide a link to the Creative Commons license, and indicate if changes were made.

\section{References}

1. World Health Organization. The atlas of heart disease and stroke. 2014. http://www.who.int/cardiovascular_diseases/resources/atlas/ en/. Accessed 26 May 2014.

2. Cho JR, Rollini F, Franchi F, et al. Unmet needs in the management of acute myocardial infarction: role of novel proteaseactivated receptor-1 antagonist vorapaxar. Vasc Health Risk Manag. 2014;10:177-88.

3. Versteeg HH, Heemskerk JWM, Levi M, et al. New fundamentals in hemostasis. Physiol Rev. 2013;93:327-58.

4. Tricoci $P$, Huang Z, Held C, et al. Thrombin-receptor antagonist vorapaxar in acute coronary syndromes. $N$ Engl $\mathrm{J}$ Med. 2011;366(1):20-33.

5. Morrow DA, Braunwald E, Bonaca MP, et al. Vorapaxar in the secondary prevention of atherothrombotic events. N Engl J Med. 2012;366(15):1404-13.

6. Ossovskaya VS, Bunnett NW. Protease-activated receptors: contribution to physiology and disease. Physiol Rev. 2004;84(2): 579-621.

7. Angiolillo DJ, Capranzano P. Pharmacology of emerging novel platelet inhibitors. Am Heart J. 2008;156(2):10S-5S
8. Coughlin SR. Protease-activated receptors in hemostasis, thrombosis and vascular biology. J Thromb Haemostasis. 2005;3(8):1800-14.

9. Shah R. Protease-activated receptors in cardiovascular health and diseases. Am Heart J. 2009;157(2):253-62.

10. Angiolillo DJ, Capodanno D, Goto S. Platelet thrombin receptor antagonism and atherothrombosis. Eur Heart J. 2010;31:17-28.

11. Chackalamannil S, Wang Y, Greenlee WJ, et al. Discovery of a novel, orally active himbacine-based thrombin receptor antagonist (SCH 530348) with potent antiplatelet activity. J Med Chem. 2008;51:3061-4.

12. Storey RF, Kotha J, Smyth SS, et al. Effects of vorapaxar on platelet reactivity and biomarker expression in non-ST-elevation acute coronary syndromes. Thromb Haemostasis. 2014;111(5):883-91.

13. Kosoglou T, Reyderman L, Tiessen RG, et al. Pharmacodynamics and pharmacokinetics of the novel PAR-1 antagonist vorapaxar (formerly SCH 530348) in healthy subjects. Eur J Clin Pharmacol. 2012;68(3):249-58.

14. Behm MO, Kosoglou T, Miltenburg AMM. The absence of a clinically significant effect of food on the single dose pharmacokinetics of vorapaxar, a PAR-1 antagonist, in healthy adult subjects. Clin Pharmacol Drug Dev. 2013;2(4):310-5.

15. Kosoglou T, Reyderman L, Tseng J, et al. Effect of food, antacid, and age on the pharmacokinetics of the oral thrombin receptor antagonist vorapaxar (SCH 530348) in healthy volunteers. Clin Pharmacol Drug Dev. 2013;2(3):223-30.

16. Kosoglou T, Kraft WK, Kumar B, et al. Pharmacokinetics and pharmacodynamics of the novel PAR-1 antagonist vorapaxar with end-stage renal disease. Eur J Clin Pharmacol. 2012;68(7):1049-56.

17. Statkevich P, Kosoglou T, Preston RA, et al. Pharmacokinetics of the novel PAR-1 antagonist vorapaxar in patients with hepatic impairment. Eur J Clin Pharmacol. 2012;68(11):1501-8.

18. Kosoglou T, Zhu Y, Xuan F, et al. Vorapaxar, an oral PAR-1 antagonist, does not affect the pharmacokinetics and pharmacodynamics of warfarin. Eur J Clin Pharmacol. 2012;68(11):1509-16.

19. Ghosal A, Lu X, Penner N, et al. Identification of human liver cytochrome P450 enzymes involved in the metabolism of SCH 530348 (vorapaxar), a potent oral thrombin protease-activated receptor 1 antagonist. Drug Metab Dispos. 2011;39(1):30-8.

20. Product information. Zontivity (vorapaxar) tablet. 2014; Whitehouse Station, NJ: Merck \& Co.

21. Kosoglou T, Zhu Y, Statkevich P, et al. The influence of multiple-dose vorapaxar, an oral PAR-1 receptor antagonist, on the single-dose pharmacokinetics and pharmacodynamics of digoxin. Clin Pharmacol Drug Dev. 2013;2(1):90-8.

22. Kosoglou T, Statkevich P, Kumar B, et al. The effect of multiple doses of ketoconazole or rifampin on the single- and multipledose pharmacokinetics of vorapaxar. J Clin Pharmacol. 2013;53(5):540-9.

23. Cornel J, Tricoci P, Lokhnygina Y, et al. Glycoprotein IIb/IIa receptor inhibitors in combination with vorapaxar, a platelet thrombin receptor antagonist, among patients with son-ST-segment elevation acute coronary syndromes (from the TRACER trial). Am J Cardiol. 2015;115:1325-32.

24. TRACER Executive and Steering Committees. The Thrombin Receptor Antagonist for Clinical Event Reduction in acute coronary syndrome (TRA-CER) trial: study design and rationale. Am Heart J. 2009;158(3):327-34.

25. Morrow DA, Scirica BM, Fox KAA, et al. Evaluation of a novel antiplatelet agent for secondary precention in patients with a history of atherosclerotic disease: design and rationale for the Thrombin-Receptor Antagonist in Secondary Prevention of 
Atherothrombotic Ischemic Events (TRA $2^{\circ} \mathrm{P}$ )-TIMI 50 trial. Am Heart J. 2009;158(3):335-41.

26. Tricoci P, Huang Z, Van de Werf F, et al. Net clinical benefit of vorapaxar in NSTE ACS: Role of ischemic and bleeding risk stratification. Circulation. 2012;126:A19049.

27. Sharma A, Helft G, Garg A, et al. Safety and efficacy of vorapaxar in secondary prevention of atherosclerotic disease: a metaanalysis of randomized control trials. Int J Cardiol. 2016; doi:10. 1016/j.ijcard.2016.10.088.

28. University of Florida and Merck Sharp \& Dohme Corp. Vorapaxar in patients with prior myocardial infarction treated with prasugrel and ticagrelor (VORA-PRATIC). 2015. https:// clinicaltrials.gov/ct2/show/NCT02545933?term=vorapaxar\&rank $=1$. Accessed 10 June 2016 .

29. Hiatt WR. Vorapaxar prevents progression of peripheral artery disease. Nat Rev Cardiol. 2013;10(7):367-8.

30. Bonaca MP, Scirica B, Creager MA, et al. Vorapaxar in patients with peripheral artery disease: results from TRA $2^{\circ} \mathrm{P}$-TIMI 50 . Circulation. 2013;127(14):1522-9.

31. Jones WS, Tricoci P, Huang Z, et al. Vorapaxar in patients with peripheral artery disease and acute coronary syndrome: insights from Thrombin Receptor Antagonist for Clinical Event Reduction in Acute Coronary Syndrome (TRACER). Am Heart J. 2014;168(4):588-96.

32. University of Florida and Merck Sharp \& Dohme Corp. Excellence In Peripheral Artery Disease Thrombin Receptor Antagonist Intervention in Claudication Evaluation (XLPAD-TRACE trial) (ALPADTRACE). 2015. https://clinicaltrials.gov/ct2/show/ NCT02660866?term $=$ vorapaxar\&rank $=12$. Accessed 10 June 2016.

33. Cavender MA, Scirica B, Bonaca MP, et al. Vorapaxar in patients with diabetes and prior MI: findings from the TRA 2P-TIMI 50 trial. Circulation. 2013;128(22 suppl):A10398.

34. Cavender MA, Scirica B, Bonaca MP, et al. Vorapaxar in patients with diabetes mellitus and previous myocardial infarction: findings from the Thrombin Receptor Antagonist in Secondary
Prevention of Atherothrombotic Ischemic Events-TIMI 50 Trial. Circulation. 2015;131(12):1047-53.

35. University of Florida and Merck Sharp \& Dohme Corp. Vorapaxar as an add-on antiplatelet therapy in post myocardial infarction patients with and without diabetes mellitus (OPTIMUS-5). 2015. https://clinicaltrials.gov/ct2/show/NCT0254865 0?term=vorapaxar\&rank=2. Accessed 10 June 2016.

36. Verrier ED, Boyle EM Jr. Endothelial cell injury in cardiovascular surgery. Ann Thoracic Surg. 1996;62(3):915-22.

37. Whellan DJ, Tricoci P, Chen E, et al. Vorapaxar in acute coronary syndrome patients undergoing coronary artery bypass graft surgery: subgroup analysis from the TRACER trial (Thrombin Receptor Antagonist for Clinical Event Reduction in Acute Coronary Syndrome). J Am Coll Cardiol. 2014;63(11):1048-57.

38. Morrow DA, Alberts MJ, Mohr JP, et al. Efficacy and safety of vorapaxar in patients with prior ischemic stroke. J Am Heart Assoc. 2013;44(3):691-8.

39. Bonaca MP, Scirica BM, Braunwald E, et al. New ischemic stroke and outcomes with vorapaxar versus placebo: results from the TRA $2^{\circ}$ P-TIMI 50 trial. J Am Coll Cardiol. 2014;64(22):2318-26.

40. Tricoci P, Huang Z, Van de Werf F, et al. Vorapaxar with and without thienopyridine use in acute coronary syndromes: results from the TRACER study. Eur Heart J. 2012;33(suppl):495-6.

41. Tricoci P, Lokhnygina Y, Huang Z, et al. Vorapaxar with or without clopidogrel after non-ST-segment elevation acute coronary syndromes: results from the Thrombin Receptor Antagonist for Clinical Event Reduction in Acute Coronary Syndrome trial. Am Heart J. 2014;168(6):869-77.e1.

42. Bonaca MP, Scirica B, Braunwald E, et al. Efficacy of vorapaxar is not modified by thienopyridine therapy: results from TRA $2{ }^{\circ} \mathrm{P}$ TIMI 50 trial. Circulation. 2012;126(21 suppl):A18595.

43. Bohula EA, Aylward PE, Bonaca MP, et al. Efficacy and safety of vorapaxar with and without a thienopyridine for secondary prevention in patients with previous myocardial infarction and no history of stroke or transient ischemic attack: results from TRA $2^{\circ}$ P-TIMI 50. Circulation. 2015;132(20):1871-9. 